gastroenterology, current standard textbooks now being of substantial mass or multivolume status.

In common with the other subject volumes in this series, it is aimed at doctors in training (both generalists and specialists), but would be equally valuable to other professionals working within the field of gastroenterology, such as nurses, physicians assistants, medical care practitioners, etc.

The authors are a small group of respected clinical gastroenterologists, including a trainee. The composition and modest size of the writing group has allowed a practical and relevant approach and a consistency of writing and presentation style, often lacking in large multi-author texts.

The objective of the book is to provide clear knowledge of guidance that would be of use in the emergency and endoscopy rooms, the ward round, and the clinic. Given the massive knowledge base informing this modest sized volume (483 pages, weight $0.9 \mathrm{~kg}$ ), it is not surprising that the authors have made extensive use of bulleted lists, tables, and diagrams. There are a useful number of imaging studies, often accompanied by a well labelled explanatory line drawing. What is not included is an extensive balanced presentation of the prevailing issues under debate, addition of which would have defeated the primary aim of producing a practical and portable book. Those readers with interests in the debates will have to look to the current journals and conferences. A reading list is provided, rather than comprehensive referencing of the text. This will undoubtedly cause occasional frustration, but referencing may have added $20-30 \%$ to the size of the book.

The book is well laid out and it is easy to navigate to specific topics, with individual section contents pages, which I found particularly helpful. The first section, "alimentary emergencies", seems particularly laid out with the admitting registrar in mind, with a good starting plan with cross referencing to further information in the rest of the book about detailed disease management for subsequent browsing when time allows.

Have the authors succeeded in their objectives? My own view is yes. Where do I see this book sitting? Certainly not in pockets; white coats have largely disappeared and, despite the book's title, an average outfit could not accommodate this tome. I envisage a great value for it as a resource for general medical examination study, and I can imagine it sitting well thumbed in junior doctor's offices and near patient areas, such as the emergency room and the medical ward. Rather tellingly, my husband, who runs an intensive care unit, has just absconded with my review copy.

P Neild

\section{Clinical Gastroenterology and Hepatology}

Edited by W M Weinstein, C J Hawkey, J Bosch. London: Elsevier Mosby, 2005, pp 1191. ISBN 0323027512

"We must go beyond textbooks, go out into the bypaths and untrodden depths of the wilderness and travel and explore and tell the world the glories of our journey" Professor John Hope Franklin.

However, textbooks are changing too Electronic editions, online extensions, PDA downloadable versions, online updates, and enhancements such as video clips have created a fusion of textbooks and the worldwide web that is changing the experience and expectations of the readership. The emerging pressures of revalidation and recertification of doctors via formal testing is also creating a new demand for practical and concise textbooks. It is now possible to travel places with a textbook and a computer.

At over 1000 pages, this textbook can hardly be labelled concise although the layout is extremely well organised. It uses all of the electronic enhancements mentioned above and is profusely illustrated with highlighted tables and message boxes. There are four colour coded sections: symptoms, syndromes and scenarios, diseases of the gut and liver, primer of diagnostic methods, and primer of treatments.

The four sections create challenges in preventing overlap and repetitions. For example, heartburn is covered in symptoms section and oesophageal diseases section, with repetitions in diagnostic tests of motility and functional tests and the treatment sections. Overall, however, the structure works reasonably well and the first two sections are the strongest. There are superb chapters such as analysis of diarrhoea, but also chapters such as functional gastrointestinal disease which simply lists a succession of tables. Some of the chapters in the initial section also have useful internet sources of information for patients and doctors. The symptoms, syndromes, and scenarios ignore an increasingly large proportion of health care seekers who wish to avoid risks by screening, although asymptomatic.

Diseases of the gut and liver are organised roughly in anatomical and conventional order but cover the entire breadth of gastrointestinal and liver disorders with a superb collection of splendidly illustrated chapters. Some, but not all, of the chapters are state of the art, with particularly strong coverage of gastric malignancies and colorectal cancer. A chapter on other gastrointestinal tumours misses opportunities for illustrations and could have been easily merged with a previous chapter on gastrointestinal stromal tumours and carcinoid tumours. Motility disorders are well covered, including a very well balanced chapter on irritable bowel syndrome.

In the primer of diagnostic methods, there are some excellent chapters on endoscopic techniques, but given the profusion of endoscopy textbooks, it may be less useful to the readership. However, virtual endoscopy and the PET chapters are well written and illustrated. Novel endoscopic imaging modalities are covered somewhat too concisely. In the primer of treatments, the chapter on drugs used in gastrointestinal and liver diseases is mostly repetition and redundant, but the nutritional assessment and management sections are strong.

The authorship is international and a refreshing number of "rising stars" are represented. The index is comprehensive. Overall, this is a welcome addition to the wide selection of textbooks available to gastroenterologists and will be useful to both trainees and experienced clinicians. It fulfils the definition of a good book.........

"That is a good book which is opened with expectation and closed in profit" Amos Bronson Alcott.

S Ghosh

\section{NOTICE OF WITHDRAWL}

doi: 10.1136/gut.2006.069237

Y S Guan, Y Liu, X P Zhou, X Li, Q He, and L Sun. p53 gene (Gendicine) and embolisation overcame recurrent hepatocellular carcinoma. Gut 2005;54:1318-19.

This case report has been withdrawn because it has already been published in the World Journal of Gastroenterology 2005;11:38035. It was submitted to Gut in error by the first author, who was unaware of the prior publication and apologises for his mistake.

\section{CORRECTION}

doi: 10.1136/gut.2005.081794corr l

Ogata $H$, Matsui $T$, Nakamura $M$, et al. A randomised dose finding study of oral tacrolimus (FK506) therapy in refractory ulcerative colitis. Gut 2006;55:1255-62.

We wish to clarify the initial total daily tacrolimus dose was $0.05 \mathrm{mg} / \mathrm{kg}$ given as $0.025 \mathrm{mg} / \mathrm{kg}$ twice daily not $0.05 \mathrm{mg} / \mathrm{kg}$ twice daily as stated in the abstract and text. 